\title{
Cosmological Constant in Coherent Quantum Gravity
}

\author{
Craig Hogan \\ University of Chicago and Fermilab
}

It is argued that quantum states of geometry, like those of particles, should be coherent on light cones of any size. An exact classical solution, the gravitational shock wave of a relativistic point particle, is used to estimate gravitational drag from coherent energy flows, and the expected gravitational effect of virtual transverse vacuum energy fluctuations on surfaces of causal diamonds. It is proposed that the appropriately spacetime-averaged gravitational effect of the Standard Model vacuum state leads to the observed small nonzero value of the cosmological constant, dominated by gravitational drag of virtual gluonic strings at the strong interaction scale. 
According to the Standard Model of particles and fields, the vacuum is not empty, but is full of fluctuating quantum fields. A naïve estimate suggests that their gravity is repulsive, and ought to accelerate the cosmic expansion like a cosmological constant $\Lambda=8 \pi G \rho_{\text {vac }}$, where the density $\rho_{\text {vac }} \sim p_{\text {max }}^{4} / c \hbar^{3}$ depends on the maximum virtual momentum $p_{\max }$ of the fluctuating modes. A cutoff at the Planck scale of quantum gravity, $p_{\max } \sim m_{P} c \equiv \sqrt{\hbar c^{3} / G}$, leads to a value $\Lambda \sim c^{5} / \hbar G \equiv t_{P}^{-2}$, about 122 orders of magnitude larger than the observed value. Clearly, this simple calculation is missing something important about the gravitational effect of virtual quanta 1 .

In this essay, it is suggested that the missing ingredient is the quantum coherence of geometry, the nonlocal quantum nature of gravity at separations much larger than the Planck length. Nobody knows exactly how this works, but it is widely suspected that significant physical effects of quantum gravity are not confined to the Planck scale 22 7], and may even produce measurable macroscopic fluctuations of causal structure 8 - 12. We sketch here a calculation that shows how geometrical quantum coherence could profoundly change the spacetime-averaged gravitational response to quantum fluctuations of the standard quantum vacuum, and explain a cosmological constant with the observed value.

To start with, recall that a quantum particle exists nonlocally, that is, without a definite relationship to space and time. A particle localized in space is in a state with delocalized momenta in all directions, which means that its location at another time is indeterminate. A particle traveling in a definite direction exists with no definite location in the normal plane. Since gravity and space-time geometry depend on mass-energy in an indeterminate superposition of locations, the quantum nonlocality, indeterminacy and coherence of matter states must somehow extend to quantum states of gravity and space-time geometry on all scales [13, 14.

Physical consequences of geometrical quantum coherence follow from correspondence with classical gravity. Consider a pair of photons emitted in opposite directions by annihilation of a positronium atom. Their directions are exactly opposite to each other in the frame of the emitting particle, but the axis is indeterminate: the wave function is a thin shell, a superposition of all directions. The direction of the photons' axis can be indeterminate for an indefinite length of time, extending everywhere on the light cone from the emission event. The causal structure remains in a superposition until the photon state "collapses" onto a particular axis. A consistent, coherent quantum geometry has to match the active gravity of the photonic energy, wherever it ends up. [15]

Gravity can be consistent with the outcome of any particle experiment if the light cone is itself a coherent quantum object, like the wave function of the photons. In that case, a measurement nonlocally affects the causal configuration of an extended region of space and time; the particles entangle with the space-time, in much the same way that Schrödinger's decayed atom entangled with his macroscopic, poisoned cat. Quantum space-time is then fundamentally discontinuous, nonlocal and discrete. Classical space and time emerge as a statistical average of many elements, similar to the way a quasi-continuous fluid emerges from a discrete molecular system [16 18 .

To quantify the physical effect of geometrical coherence, consider first the classical gravitational shock wave of a localized relativistic point particle in flat space-time [19]21. A particle with momentum $p$ and impact parameter $x_{\perp}$ creates a coherent displacement everywhere on a conical light sheet (Fig. 11),

$$
\delta u=4 G p c^{-3} .
$$

This displacement represents a real, physical distortion of causal structure; the motion of the particle "drags" the space-time along with it. The shock creates an axially symmetric distortion of the causal structure in the directions normal to the particle axis.

This classical solution allows us to construct simple examples of coherent quantum states of geometry associated with coherent energy flows of particle states. For a state like our positronium annihilation, with two counterpropagating particles in a superposition of orientations (Fig. 2), the space-time is also in a superposition of spacelike distortions. The orientation of the distortion is entangled with the particle axis, and extended over space and time. A collapse onto a definite particle trajectory leads to collapse of an extended causal diamond, with a dislocation in the normal directions, wherever they end up. For two coplanar classical particle trajectories $A, B$ with $\vec{p}_{A}=-\vec{p}_{B}$ that do not pass through a point, but are separated by an impact parameter $x_{A B}$ as they pass by, gravitational drag creates a rotational impulse, a "twist" of the local inertial frame. Setting aside geometrical factors of the order of unity, the angular velocity of the twist normal to the plane is about

$$
\omega \sim c \delta u_{A B} / x_{A B}^{2} \sim G p_{A B} / c^{2} x_{A B}^{2},
$$

where $p_{A B}=\left|\vec{p}_{A \perp}-\vec{p}_{B \perp}\right|$ denotes the transverse momentum difference of the particles.

We can use these states to estimate the mean gravitational effect of quantum field vacuum fluctuations on a coherent geometrical state. The mean rotation $\langle\omega\rangle$ of the inertial frame vanishes, but there are nonvanishing fluctuations, determined by the mean square transverse momentum flow on light cones :

$$
\left\langle\omega^{2}\right\rangle \sim G^{2} c^{-4}\left\langle p_{A B}^{2} / x_{A B}^{4}\right\rangle_{C D},
$$




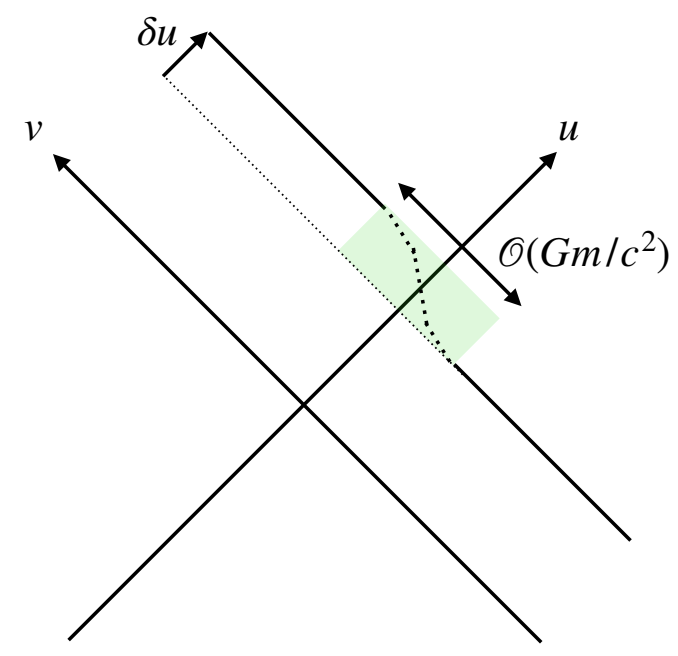

FIG. 1. The classical gravitational dislocation of causal structure from a very fast-moving point mass $m$ [19] 21 . In null coordinates $u, v$ on the light cone of the particle moving in the $u$ direction with momentum $p>>m c$, the gravitational drag of the particle (Eq. 1) creates a displacement $\delta u$ that varies on opposite sides of the trajectory. The physical displacement is axially symmetric around the particle trajectory and independent of the impact parameter of the particle, $x_{\perp}$.
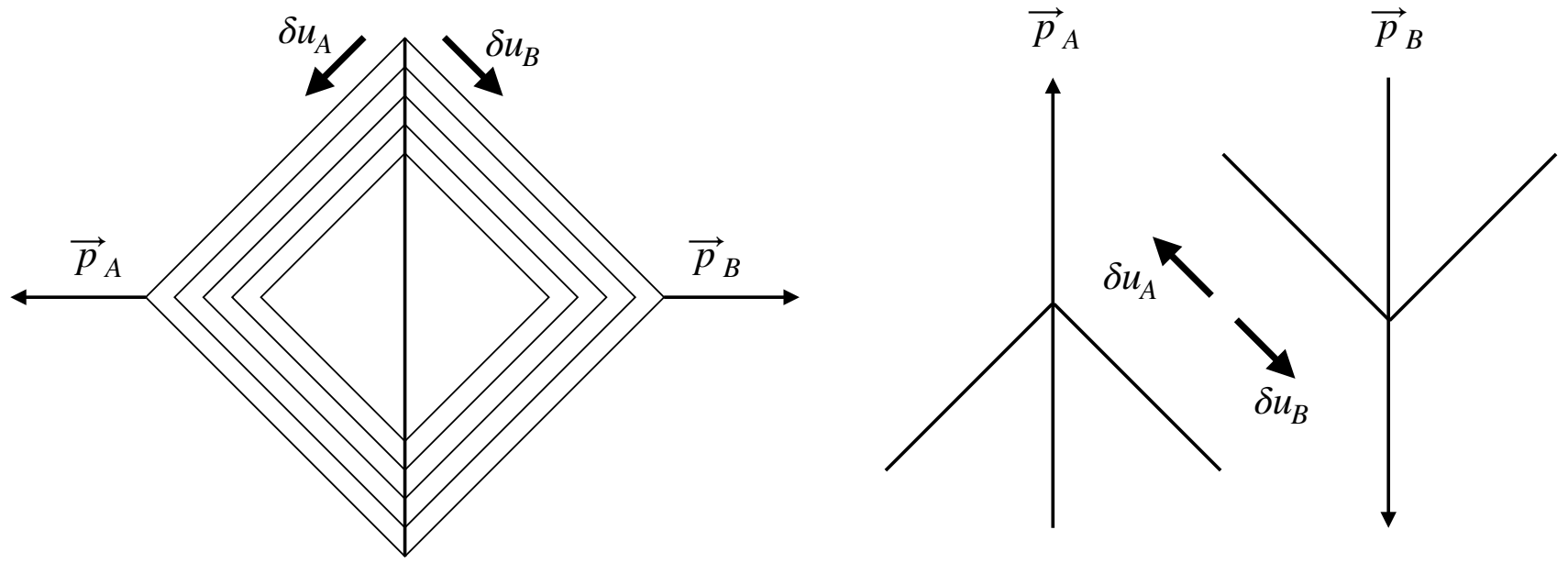

FIG. 2. Coherent spatial patterns of conical shock waves from counterpropagating particles, with perturbations $\delta u_{A}, \delta u_{B}$ due to frame dragging. At left, an inwards shock forms from a pair created at the origin by annihilation, shown at a series of successive times. The orientation of a pair of entangled particles can remain indeterminate to arbitrarily large scales; causal structure is placed in a superposition on the whole series of causal diamond surfaces far from the particles, with a compression in the plane normal to the axis. At right, two particles travel transversely to an observer in between. In a quantum vacuum, a nonlocally correlated state with fluctuating angular momentum can cause tiny rotational fluctuations by dragging the local inertial frame (Eq. 3), and a net outwards acceleration of geodesics (Eq. 4).

where \langle\rangle$_{C D}$ denotes an expectation value over invariant spherical causal diamond surfaces for a world line interval, and $p_{A B}$ denotes the net transverse momentum flow at opposite points $A$ and $B$ on each surface, with spacelike separation $x_{A B}$.

The sign of the mean square $\left\langle\omega^{2}\right\rangle$ is always positive, so the fluctuating impulses systematically accumulate outwards displacements of causally-defined surfaces. Let us suppose that these exotic fluctuations of the inertial frame have the same effect on the radial component of geodesics in the time-averaged, emergent geometry as classical rotation, 
that is to say, a mean centrifugal acceleration $\ddot{r}$ proportional to separation $r$ :

$$
\langle\ddot{r} / r\rangle=\left\langle\omega^{2}\right\rangle \text {. }
$$

The time-averaged physical effect on freely falling trajectories is then the same as a cosmological constant $\Lambda=3\left\langle\omega^{2}\right\rangle$ : the gravity of fluctuating twists in the virtual vacuum pulls the emergent space-time apart.

The gravitational effect from the particle vacuum with this kind of coherent quantum average over causal diamonds is much less than the naïve calculation outlined above based on uncorrelated field modes. The sum of linear dislocations from virtual pairs in all directions averages to zero, so approximately-pointlike virtual particle states produce approximately-vanishing gravitational fluctuations. However, the gravitational effect does not exactly vanish. In the Standard Model, the pointlike-particle approximation breaks down from the strong self-coupling of gluons 22, 23. at the QCD energy scale $m_{Q} c^{2}$, which largely determines the masses of protons, neutrons, and atoms, as well as the range of nuclear interactions. Coherent gluonic fluctuations at this scale include significant spacelike correlations of transverse momentum.

In a coherent quantum geometry, these momentum fluctuations produce a tiny bit of gravitational repulsion. The gravitational effect can be estimated from Eq. (3), with $p_{A B} / c \sim \hbar / x_{A B} c \sim m_{Q}$ :

$$
\left\langle\omega^{2}\right\rangle \sim G^{2} m_{Q}^{6} c^{2} / \hbar^{4} \sim t_{P}^{-2}\left(m_{Q} / m_{P}\right)^{6}
$$

where $t_{P} \equiv \sqrt{\hbar G / c^{5}}=0.54 \times 10^{-43}$ sec and $m_{P} \equiv \sqrt{\hbar c / G}=1.22 \times 10^{19} \mathrm{GeV} / c^{2}$. The measured cosmic acceleration[24]

$$
\sqrt{\left\langle\omega^{2}\right\rangle}=\sqrt{\Lambda / 3} \approx 1.0 \times 10^{-61} t_{P}^{-1}
$$

would be produced by vacuum fluctuations with mass on the order of

$$
m_{Q} \sim\left(\sqrt{\left\langle\omega^{2}\right\rangle} t_{P}\right)^{1 / 3} m_{P} \sim 0.5 \times 10^{-20} m_{P} \sim 60 \mathrm{MeV} / c^{2}
$$

This rough estimate is remarkably close to the actual scale of strong interactions, as measured by the masses of their lowest-energy real particle states, the pions [24]:

$$
m_{\pi^{0}}=135 \mathrm{MeV} / c^{2}
$$

Like pions and other mesons, vacuum fluctuations on the nuclear scale can be visualized not as virtual pointlike particles, but as spatially extended strings of gluon condensate with quarks at the ends. The bulk of the stressenergy is in the gluonic string, whose stress-energy is dominated by negative pressure or tension. The gravity of a string, unlike that of a point particle, is repulsive, so stringlike virtual-gluon states naturally lead to a small cosmic acceleration, with a value similar to that observed. 25]

This scenario roughly accounts for the well-known puzzling coincidence 26 28] of cosmic acceleration (Eq. 60 with the rate of stellar evolution, which depend on the same power of the small number $m_{Q} / m_{P}$. The origin of this small dimensionless number (and the relative weakness of gravity) is well understood in the Standard Model, from the logarithmic running of the strong coupling constant with energy scale 22].

The departure of the cosmological constant from zero in this picture is a low-energy, infrared phenomenon. Its physical value depends only on Standard Model fields, and a standard gravitational response to an appropriate average over their quantum stress-energy fluctuations. The expected gravitational effect of vacuum fluctuations (Eq. 3 depends on nonlocal spacelike correlations of QCD fields, which can in principle be calculated on a lattice, as nucleon masses are 23, 24. It does not depend on detailed properties of Planck-scale physics, except for simple symmetries of coherent geometrical states on scales $>t_{P}$.

\section{ACKNOWLEDGMENTS}

This work was supported by the Department of Energy at Fermilab under Contract No. DE-AC02-07CH11359. 
[1] Steven Weinberg, "The Cosmological Constant Problem," Rev. Mod. Phys. 61, 1-23 (1989)

[2] A. G. Cohen, D. B. Kaplan, and A. E. Nelson, "Effective field theory, black holes, and the cosmological constant," Phys. Rev. Lett. 82, 4971 (1999).

[3] Shinsei Ryu and Tadashi Takayanagi, "Holographic derivation of entanglement entropy from AdS/CFT," Phys. Rev. Lett. 96, 181602 (2006), arXiv:hep-th/0603001 [hep-th].

[4] Gerard 't Hooft, "Virtual black holes and space-time structure," Foundations of Physics 48, 1134-1149 (2018)

[5] Daniel Carney, Philip C E Stamp, and Jacob M Taylor, "Tabletop experiments for quantum gravity: a user's manual," Classical and Quantum Gravity 36, 034001 (2019)

[6] Steven B. Giddings, "Black holes in the quantum universe," Proceedings, Topological avatars of new physics: London, United Kingdom, March 4-5, 2019, Phil. Trans. Roy. Soc. Lond. A377, 20190029 (2019), arXiv:1905.08807 [hep-th]

[7] Erik Verlinde and Kathryn M. Zurek, "Spacetime Fluctuations in AdS/CFT," (2019), arXiv:1911.02018 [hep-th].

[8] A. Chou, H. Glass, H. R. Gustafson, C. J. Hogan, B. L. Kamai, O. Kwon, R. Lanza, L. McCuller, S. S. Meyer, J. Richardson, C. Stoughton, R. Tomlin, and R. Weiss (Holometer Collaboration), "Interferometric Constraints on Quantum Geometrical Shear Noise Correlations," Class. Quant. Grav. 34, 165005 (2017), arXiv:1703.08503 [gr-qc].

[9] Erik P. Verlinde and Kathryn M. Zurek, "Observational Signatures of Quantum Gravity in Interferometers," (2019), arXiv:1902.08207 [gr-qc].

[10] Craig Hogan, "Nonlocal entanglement and directional correlations of primordial perturbations on the inflationary horizon," Phys. Rev. D 99, 063531 (2019).

[11] Craig J Hogan, "Pattern of perturbations from a coherent quantum inflationary horizon," Classical and Quantum Gravity (2020), arXiv:1908.07033 [astro-ph.CO]

[12] Ray Hagimoto, Craig Hogan, Collin Lewin, and Stephan S. Meyer, "Symmetries of CMB temperature correlation at large angular separations," The Astrophysical Journal 888, L29 (2020)

[13] John Archibald Wheeler, "Problems and prospects in elementary particle research," Proceedings of the American Philosophical Society 90, 36-47 (1946)

[14] John A. Wheeler, "Geometrodynamics and the problem of motion," Rev. Mod. Phys. 33, 63-78 (1961).

[15] This hypothesis differs physically from the quantized linear field theory of gravity, where quantum coherence is attached to the amplitude of a plane wave mode whose space-time configuration is defined by an unquantized background geometry. Modes that localize a point particle in the plane normal to its axis of propagation are not independent in coherent quantum geometry, since they are entangled with spherical surfaces of causal structure in all directions around an event. As shown here, this radically changes the space-time-averaged gravitational effect of vacuum fluctuations in energy-momentum.

[16] T. Jacobson, "Thermodynamics of Spacetime: The Einstein Equation of State," Phys. Rev. Lett. 75, 1260 (1995).

[17] T. Jacobson, "Entanglement Equilibrium and the Einstein Equation," Phys. Rev. Lett. 116, 201101 (2016), arXiv:1505.04753 [gr-qc].

[18] T. Padmanabhan, "General Relativity from a Thermodynamic Perspective," Gen. Rel. Grav. 46, 1673 (2014), arXiv: 1312.3253 [gr-qc]

[19] P. C. Aichelburg and R. U. Sexl, "On the gravitational field of a massless particle," General Relativity and Gravitation 2, 303-312 (1971)

[20] Tevian Dray and Gerard 't Hooft, "The gravitational shock wave of a massless particle," Nuclear Physics B 253, 173 $188(1985)$

[21] Gerard 't Hooft, "Black hole unitarity and antipodal entanglement," Found. Phys. 46, 1185-1198 (2016), arXiv:1601.03447 $[\mathrm{gr}-\mathrm{qc}]$.

[22] F. Wilczek, "Quantum field theory," Rev. Mod. Phys. 71, S85 (1999).

[23] Frank Wilczek, "QCD made simple," Phys. Today 53N8, 22-28 (2000)

[24] M. Tanabashi et al. (Particle Data Group), "Review of Particle Physics," Phys. Rev. D98, 030001 (2018)

[25] Similarly, in a classical thermodynamic view, the adiabatic "work" done on virtual gluonic strings slightly stretched by gravitational repulsion is the source of the new vacuum "energy" in the expanded space. This microscopic view of the cosmological constant has a complementary holographic interpretation in terms of geometrical information: the entanglement entropy of the horizon matches the independent field degrees of freedom below the QCD scale 28. It differs physically from other recent microscopic models [29, 30] seeking to connect the cosmological constant with standard model vacuum states, based on Planck scale discreteness and electroweak scale torsion.

[26] Ya. B. Zeldovich, "The cosmological constant and the theory of elementary particles," Physics-Uspekhi 11, 381-393 (1968).

[27] Craig J. Hogan, "Why the universe is just so," Rev. Mod. Phys. 72, 1149-1161 (2000), arXiv:astro-ph/9909295 [astro-ph]

[28] C. J. Hogan, "Exotic Rotational Correlations in Quantum Geometry," Phys. Rev. D 95, 104050 (2017), arXiv:1509.07997 $[\mathrm{gr}-\mathrm{qc}]$.

[29] Alejandro Perez and Daniel Sudarsky, "Dark energy from quantum gravity discreteness," Phys. Rev. Lett. 122, 221302 (2019), arXiv:1711.05183 [gr-qc]

[30] Alejandro Perez, Daniel Sudarsky, and James D. Bjorken, "A microscopic model for an emergent cosmological constant," Int. J. Mod. Phys. D27, 1846002 (2018), arXiv:1804.07162 [gr-qc] 